\title{
DO ENGINEERS USE CONVERGENCE TO A VANISHING POINT WHEN SKETCHING?
}

\author{
Raquel Plumed \\ Dept. of Mechanical \\ Engineering and \\ Construction \\ Universitat Jaume I \\ 12071 Castellon \\ plumed@emc.uji.es
}

\author{
Pedro Company \\ Dept. of Mechanical \\ Engineering and \\ Construction \\ Universitat Jaume I \\ 12071 Castellon \\ pcompany@emc.uji.es
}

\author{
Ana Piquer \\ Dept. of Mechanical \\ Engineering and \\ Construction \\ Universitat Jaume I \\ 12071 Castellon \\ Ana.Piquer@emc.uji.es
}

\author{
Peter A.C. Varley \\ Dept. of Mechanical \\ Engineering and \\ Construction \\ Universitat Jaume I \\ 12071 Castellon \\ varley@emc.uji.es
}

\begin{abstract}
We wish to determine whether design engineers commonly use central projections (convergence of parallel lines to a vanishing point) when sketching new shapes, rather than draw physically parallel lines as parallel. This paper describes a pilot experiment carried out to determine the presence and importance of central projections. Results suggest that designers rarely use vanishing points when sketching engineering shapes. Hence, convergence can safely be ignored when designing and implementing basic artificial intelligence systems which detect perceptual cues in engineering design sketches.

Since we wish to develop an automated method for discriminating between central and parallel pictorial projections, the paper also presents a numerical analysis of our results which could be used to calibrate such a method.
\end{abstract}

\section{Introduction}

Artificial intelligence aims to mimic human intelligence, and the most interesting artificial intelligence research is that which throws light on human intelligence (see, for example, [1]). Visual perception is a complex aspect of human intelligence, and its artificial equivalent, machine vision, has been much studied.

There is a clear synergy between the studies of visual perception and machine vision: knowing as much as possible about human perception provides resources which help us to develop artificial perception methods which are intuitively correct [2], while creating artificial perception tools reminds us of what we still need to learn about human vision.

Our area of interest is creating computerbased tools for design engineers, and for this reason we wish to understand how designers make use of perceptual cues in engineering sketches.

Parallel lines are a particularly important cue, and they have two common graphical representations in pictorial projections which have survived the test of time. One, central projection (used since the $15^{\text {th }}$ Century and codified in Durer's Four Books on Measurement in 1522), is the convergence to one or more vanishing points of lines representing parallel lines in 3D space. The alternative, axonometric projection (at least as old in practice, but only codified in the $19^{\text {th }}$ Century by Farish [3]), does not use vanishing points - convergence of parallel lines is deliberately absent. A third representation, oblique projection, also deliberately avoids the use of vanishing points, and in this paper oblique sketches are generally grouped with axonometric sketches.

Clearly, each representation has advantages and disadvantages, and engineers and designers must be trained to use both. However, which representation they prefer for any particular task has not been fully investigated. As far as we know, determining whether engineers and designers commonly use convergence of parallel lines while sketching new shapes is still an unresolved question.

Such questions can only be answered by experiment. The work we present here describes a pilot experiment carried out to determine the presence and importance of vanishing points in sketches produced by engineers and designers. 
Section 2 discusses related work. Section 3 describes the hypotheses which we wish to test. Section 4 describes in detail our pilot experiment to determine whether central projections are preferred when making engineering sketches, and presents a visual analysis of the results, used to test our hypotheses. Section 5 presents a numerical analysis which could be used to calibrate an automated classification method, and Section 6 presents our preliminary conclusions.

\section{Related work}

Physiologists have studied perceptual cues and how they guide the visual perception process (e.g. [4], [5], [6]), and some correspondences between perceptual organisation in biological and artificial vision have been established (see, for example, [7]). Some computer scientists have replicated cue-based perception processes in various approaches (e.g. [8], [9], [10], [11], [12]). Even so, the current situation is that we have no complete catalogue of perceptual cues, and the exact role even of known cues is not fully understood.

In particular, the importance of central projection as a perceptual cue specific to the interpretation of engineering sketches has not been investigated experimentally. Previous studies have in general either assumed or ignored convergence as one of their simplifying assumptions. For example, Parodi el al. [13] investigating the computational time complexity of labelling polyhedral scenes, assume the presence of convergence and show that calculating vanishing points is the ratedetermining step. Sturm et al. [14] calculate vanishing points to calibrate single camera images of polyhedra, but require the user to specify manually which lines are intended to be parallel. Kanade [15] proposes a technique for recovering three dimensional shapes from a single image, based on mapping image regularities (in particular, parallelism of lines and skewed symmetry) into shape constraints.

Prats et al. [16] investigates the sketching process of designers and how they are able to obtain new shapes during sketching by the application of shape rules, but it does not consider the study of depth cues.
Wyeld [17] presents a psychology experiment directed at determining the range of variability in individuals' drawing ability and ability to read 3D images. The drawings used in the study included common perspective depth cues such shading, shadows and a ground plane. The population for this study was limited to first year undergraduate students. In contrast to Wyeld's study, we focus on one specific depth cue, but we consider a much wider range of technical drawing skill.

\section{Hypothesis}

The purpose of our experiment is to determine to what extent convergence to a vanishing point is used by designers while sketching engineering shapes. It is here that we must define designer.

For the purposes of our experiment, a product designer is someone who has received specific training in 2D and 3D geometry and the commonly-used techniques for representing 3D objects in 2D as part of a technical education.

We contrast product designers with graphic designers, who have received training in the commonly-used techniques for representing 3D objects in $2 \mathrm{D}$ as part of a non-technical (often artistic) education.

Using these definitions, we hypothesise that the technical training received by product designers influences the way they represent three dimensional parts. In particular, we hypothesise that people who are trained to think in engineering terms will generally prefer parallel (axonometric or oblique) projection (which retains the important cues to functionality) rather than perspective projection.

By contrast, we might expect that graphic designers, after being exposed to more artistic training (where how it looks is more important than what it does), may show a trend towards using convergence when making design sketches.

Therefore, we should compare the behaviour of two distinct population groups: product designers whose background is in engineering, and graphic designers whose background is artistic.

Ideally, we should also consider a third group, those who have, as yet, received no design training either as engineers or as artists. In this case, we have no reason to hypothesise a 
preference either for parallel projections or convergence.

Finally, we are interested in developing an algorithmic approach that can automatically discriminate between parallel and central projection sketches.

\section{Design of the experiment}

In this section, we describe the experiment which we designed to test our hypothesis.

The basis of our experiment is that we asked various people to draw pictures of three polyhedral solids. The solids and the accompanying instructions are described in detail in Section 4.1, and the participants in the study are described in detail in Section 4.2.

To determine the human perception of such drawings, the pictures were subjectively classified by a group of experts as central/axonometric projections and good/poor quality. The classification results are summarised in Section 4.3.

\section{1.}

\section{The questionnaire}

In designing our experiment, it is important to avoid any kind of implicit or explicit constraint or guidance on the way the task should be performed. In particular, we tried not to influence the participants either to use or not to use hidden lines, and left them free to choose the orientation of the model.

For this purpose, we produced a minimal questionnaire which avoided as far as possible any unnecessary guidance to the participants. Since a verbal task description could implicitly suggest some restrictions in the sketch process, the task description in the questionnaire took the form of a visual worked example: a photograph of a physical object and its representation as a freehand drawing.

Expanded polystyrene was chosen for the model material as surface brightness helps the observer to recognise the object's faces and edges, thereby ensuring that all participants had a good mental model of the object they were to sketch.

To avoid any hint of how parallel edges should be represented, or whether or not hidden lines should be drawn, the physical object in the worked example was a tetrahedron (which, obviously, contains no parallel lines) oriented such that all of its edges were visible. Figure 2 shows a copy of the questionnaire.

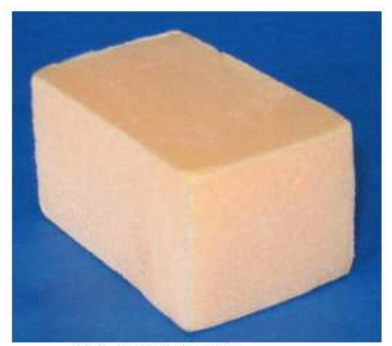

(a) First model

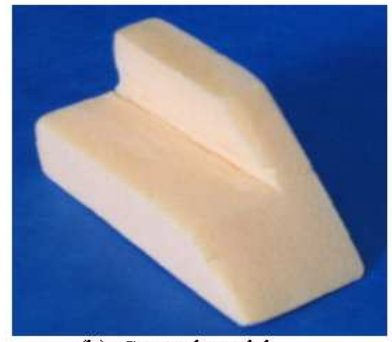

(b) Second model

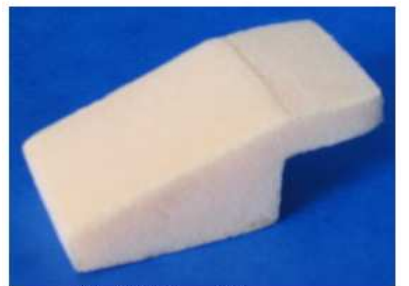

(c) Third model

Figure 1. Models used in the experiment, by rising order of difficulty: (a), (b) and (c).

Even this minimal task description contains one implicit hint: the participants are influenced to use pictorial projection, not multiview orthographic projections.

The questionnaire also included a $15 \times 11 \mathrm{~cm}$ rectangular frame for the participants to draw their own objects. This helped to ensure that sketches were of a similar size.

As part of the experimental process, we also collected personal data about the individual participants. These data did not compromise the participants' anonymity, and the answers could be useful in the analysis stage, allowing us to correlate the results with other data as studies level, studies field, sex and age. 


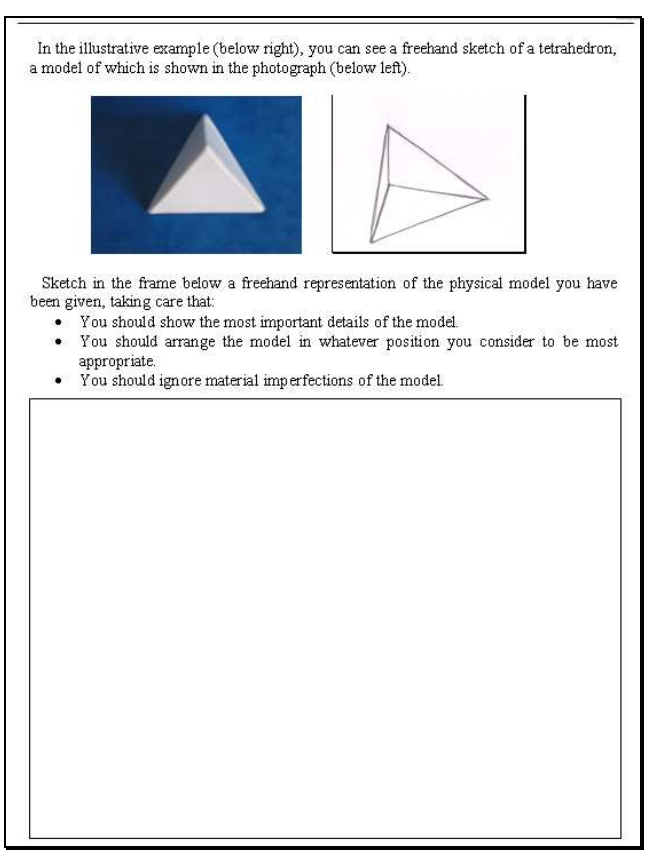

Figure 2. Questionnaire model

\section{2.}

\section{Participants}

The bulk of the population was drawn from several departments of the same university, and included industrial engineers, mechanical engineers, architects, designers and artists. The level of experience ranged from undergraduate students to professors. We also included a few participants with no technical drawing training.

A total of 147 questionnaires were returned.

$16(10.88 \%)$ were returned by participants with no university education. 2 of them $(12.5 \%)$ were filled out by people with secondary education until 16 years old. 3 of them $(18.75 \%)$ were solved by people with secondary education between 16 and 18 years old. And others 11 $(68.75 \%)$ had received secondary education with professional orientation. Their ages ranged from 27 to 66 years old. 11 were male $(68.75 \%)$ and 5 were female $(31.25 \%)$.

73 questionnaires (49.66\%) were returned by university students, of whom 58 (79.45\%) studied an engineering speciality (mechanical, industrial), $7(9.59 \%)$ studied architecture, and $8(10.96 \%)$ studied other subjects. Their ages ranged from 18 and 43 years old. 53 were male $(72.6 \%)$ and 20 were female $(27.4 \%)$.

58 questionnaires $(39.46 \%)$ were returned by participants with one or more university degree. $29(50 \%)$ graduated in engineering, $14(24.14 \%)$ graduated in architecture, $8(13.79 \%)$ had artistic training via design studies or BBAA, and 7 $(12.07 \%)$ graduated in other fields. Their ages ranged from 26 to 56 years old. 35 were male $(60.34 \%)$ and 23 were female (39.66\%).

\subsection{Human perception}

In order to determine how humans would classify the sketches, each sketch was subjectively classified by six experts (four belonging to the research team and two external experts) as: clearly axonometric; clearly central; clearly non-pictorial orthographic; uncertain; and not classifiable because of poor quality.

Next, we compared the experts' classifications, and discarded those drawings where there was disagreement (agreement means here that four or more experts chose the same classification). As a result, 20 (13.6\%) of the original 147 drawings were discarded.

We also discarded 9 sketches which were considered by all six experts as so poor quality that trying to classify their contents as central or parallel projections was pointless.

Finally, we discarded 7 sketches which were agreed as drawn using non-pictorial orthographic projections, as, showing only a single $2 \mathrm{D}$ view, these were not useful when determining how 3D objects are represented pictorially.

After discarding useless sketches, we were left with 111 valid sketches. Of these, $3(2.54 \%)$ clearly used central projection and 108 (91.53\%) clearly used axonometric projection. All 3 of those which used central projection depicted the first model (the cuboid).

In more detail:

- 71 of the valid sketches (63.96\%) were created by product designers (graduated and undergraduate students from engineering studies). All of these were classified as axonometric drawings by experts.

- 26 of the valid sketches (23.42\%) were created by graphic designers (graduated and undergraduate students of architecture, design and artistic studies). These included 
the 3 were classified by experts as perspective drawings.

- 14 of the valid sketches (12.61\%) were produced by subjects without drawing training.

From these results, we find strong support for the hypothesis that designers (both product designers and graphic designers) prefer axonometric to perspective projection. There is only weak support for the hypothesis that graphic designers are more likely than product designers to use perspective projection, and there is also only weak support for the hypothesis that model complexity influences choice of representation, with perspective projection being more likely for simple objects than for complex objects.

\section{Numerical Measurements}

In order to preserve the questionnaires, the sketched images were scanned and saved as bitmaps. This has the additional advantage that it allows us to perform numerical analyses. This numerical data could be used to calibrate automatic classification of sketches into central/axonometric, as a step towards our aim of automating interpretation of engineering sketches. This data can be found in [18].

In order to produce this numerical data, we manually vectorised the scanned images into line drawings, by identifying vertex locations and tracing new lines from vertex to vertex, as described next.

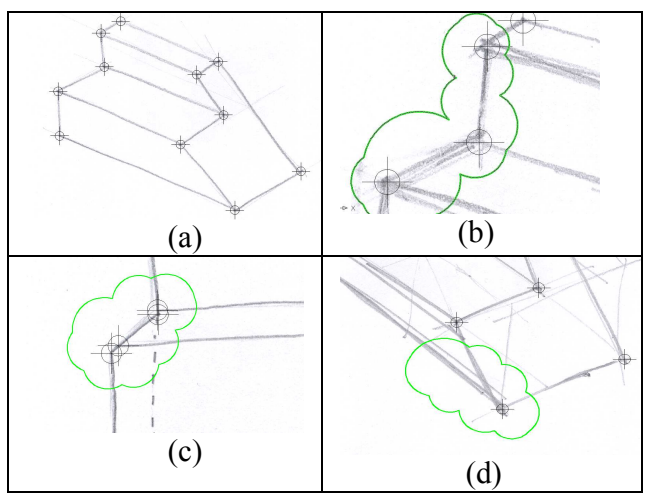

Figure 3. Vertices defined by points intersection
In most cases, vertex locations are clearly defined as junctions of two or more line segments, as shown in Figure 3 (a).

However, there were also cases in which vertex locations were not so well defined:

- Overtracing, as in Figure 3(b), results in several intersection points among several lines. In these cases, we defined the vertex location as the intersection of medial axes.

- Sometimes, as in Figure 3(c), junctions of lines which were intended to intersect at the same vertex were sufficiently separated to be considered as distinct vertices. In our processing, we merged any two vertex locations which differed by less than $3.5 \%$ of the length of the shortest line segment intersecting either vertex.

- Finally, some participants used scaffolding lines intermixed with pictorial lines, as in Figure 3(d) (scaffolding is any line or group of auxiliary lines in the sketch which is used to facilitate drawing and which does not correspond to any feature of the object). We assumed that thick lines are pictorial lines and thin lines are scaffolding, and vertex locations only occur at the intersections of pictorial lines.

Once all vertex locations were defined, we redrew pictorial lines in different colours, where each colour corresponds to a different direction (see Figure 4).

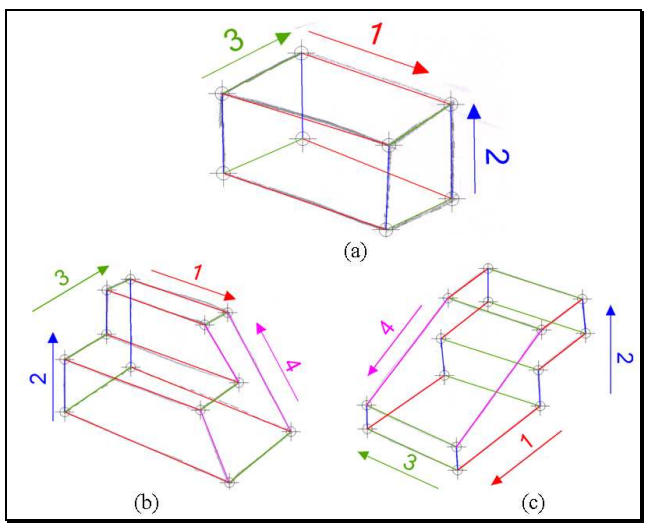

Figure 4. Line drawings

In order to facilitate later analysis, lines representing the same edges of each model were always drawn in the same colour. 
Hidden lines were also vectorised, since they portray edges of the model, but other auxiliary lines (such as reference axes and scaffolding) were discarded.

From each vectorised line drawing we extracted: (a) geometrical information provided line slopes, line lengths and coordinates of every vertex; (b) general information, corresponding to geometric information sorted by direction; and (c) drawing information: on the existence or absence of hidden lines or auxiliary lines in drawings.

We analysed these numerical results with the objective of looking for criteria which could be used to discriminate between parallel and convergent sketches. This analysis is presented in Section 5.1. The analysis produced other interesting results, which are discussed briefly in Section 5.2.

\subsection{Automatic discrimination of central style sketches}

Obviously, not all people have the same ability to create accurate freehand sketches. We cannot simply look for parallel lines to discriminate parallel projections from central projections: in reality, no lines in a freehand sketch will be perfectly parallel.

Instead, we need a calculable function which can be used to distinguish deliberate parallelism and/or convergence from involuntary mistakes made during sketching. By analysing data from the 108 valid drawings classified as axonometric, we can then estimate a threshold value for this function.

Our choice of function is angular dispersion (AD), the maximum angle value among the slope differences between each pair of lines representing parallel edges parallel of the original 3D model:

$A D=\max \left|\alpha_{i}-\alpha_{j}\right| / i=1,2, . ., n ; j=1,2, . ., n ; i \neq j$, where $n$ defines each of the set of lines belonging to a specific direction. It is intended to measure the maximum freehand sketching error which occurs when designers draw parallel lines.

Several aspects of the $\mathrm{AD}$ function were studied.

First we created histograms which show the relative frequency (in $1^{\circ}$ buckets) of $\mathrm{AD}$ for each model and direction (i.e. group of like-coloured lines). Figure 5 shows the histograms for model 1.
From the original data, we also extracted the mean values of $\mathrm{AD}$ for each model and direction (see table 1)

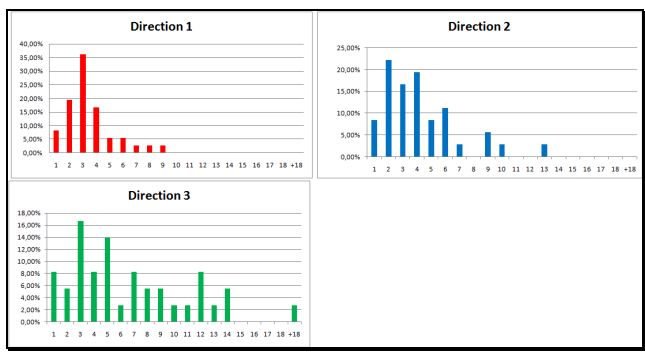

Figure 5. Histograms of relative frequency vs intervals of angular dispersion for model 1.

\begin{tabular}{|l|c|c|c|}
\hline & Model 1 & Model 2 & Model 3 \\
\hline Direction 1 & $2,98^{\circ}$ & $4,28^{\circ}$ & $7,25^{\circ}$ \\
\hline Direction 2 & $3,63^{\circ}$ & $4,77^{\circ}$ & $12,54^{\circ}$ \\
\hline Direction 3 & $6,19^{\circ}$ & $12,08^{\circ}$ & $8,65^{\circ}$ \\
\hline Direction 4 & & $5,97^{\circ}$ & $2,96^{\circ}$ \\
\hline
\end{tabular}

Table 1. The mean values of $\mathrm{AD}$ for each model

Secondly, we plotted the cumulative frequency of $\mathrm{AD}$, as shown in Figure 6. We found that, owing to the part orientation chosen by some participants, in 22 cases there was only one edge in one or more of the directions, making it impossible to perform this calculation. These 22 cases were not included in this analysis.

We obtained a mean $\mathrm{AD}$ value of $6.5^{\circ}$. Fixing a threshold value of $\mathrm{AD}=9^{\circ}$ would lead to $80.4 \%$ of directions being classified as parallel rather than converging. If we raise the threshold value to $\mathrm{AD}=13^{\circ}$, then $90.1 \%$ of directions would be classified as parallel.

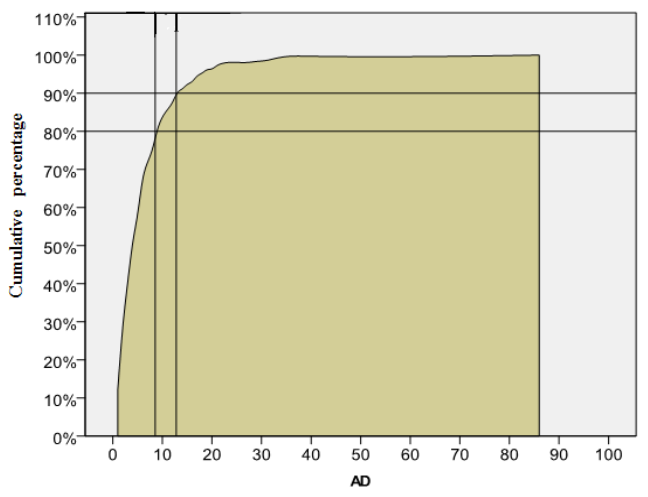

Figure 6. Cumulative Frequency of overall AD 
Analysing the sketches which were classified manually as perspective drawings, we found that the mean value of their $\mathrm{AD}$ is $21.84^{\circ}$, and the lowest value is $8.6^{\circ}$.

We therefore propose that a value of $\mathrm{AD}=8^{\circ}$ would be suitable for determining whether the designer's intention was to draw parallel lines. From the graph in Figure 6, 75.4\% of $\mathrm{AD}$ values are below this threshold.

\subsection{Other results}

Our data also allows us to investigate the preference of designers for particular direction angles in 2D. Figure 7 shows the frequency of particular angles, grouped in $4^{\circ}$ buckets. As can be seen, there is a strong preference for $90^{\circ}$ (i.e. vertical), followed by a weaker preference for $30^{\circ}$, $0^{\circ}$ and $-30^{\circ}$.

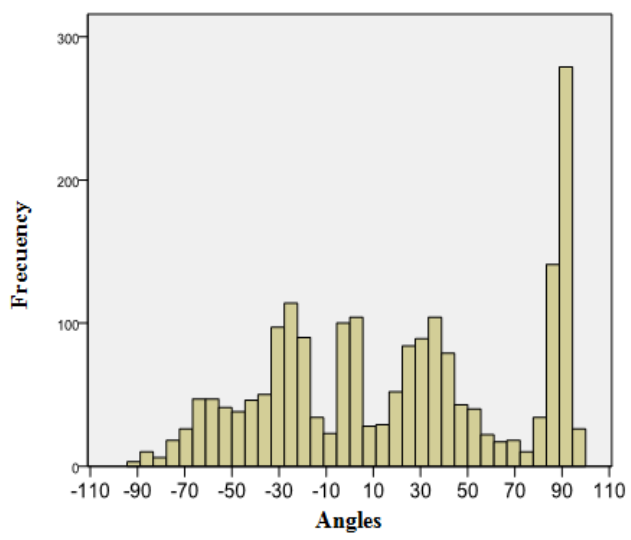

Figure 7. Frequency of angles

Earlier studies (e.g. [19]) have theorised that vertical edges of a $3 \mathrm{D}$ object will be drawn as vertical lines in 2D. The predominance of $90^{\circ}$ angles, independent of the model drawn and the type of projection used, strongly supports this theory. Verticality is a dominant direction in pictorial representation, and both product and graphic designers are able to use it with accuracy.

The frequency of $\pm 30^{\circ}$ can be attributed to them being the required angles of the two horizontal axes in isometric projection.

Finally, in creating Table 1, we observed that the largest $\mathrm{AD}$ values corresponded to short lines. Taking this further, we looked for a possible correlation between $\mathrm{AD}$ values and average lengths of each set of lines. The results are plotted in Figure 8, which shows that there is indeed some correlation between the two parameters. This study falls outside the scope of our present paper, but is something which could profitably be investigated in the future.

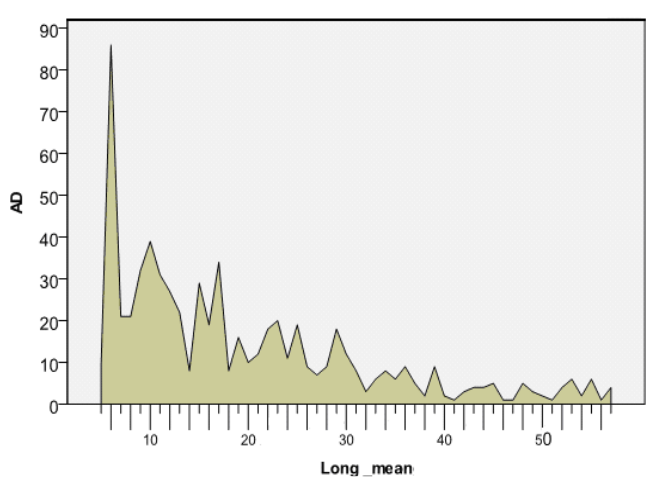

Figure 8. Diagram of dispersion points between $\mathrm{AD}$ values and average lengths.

\section{Conclusions}

From our experiment, we conclude that convergence is not an important cue for artificial perception of engineering sketches, as central projections are rarely used in engineering sketches. By contrast, parallelism is a very important cue, as parallel lines of threedimensional shapes are usually represented as parallel lines in engineering sketches.

Both product and graphic designers tend to prefer axonometric projections when sketching simple polyhedral engineering parts. There is a suggestion that this preference becomes stronger with more complex objects, but the supporting evidence is weak and this remains a matter for future research.

Automatic discrimination between central and axonometric sketches remains problematic. The fact that line are or are not parallel (to within any particular threshold value) is not a sufficiently reliable indicator of the choice between central or axonometric projection. A threshold value $\left(\mathrm{AD}=8^{\circ}\right)$ low enough to correctly identify all central projections would also lead to $24.6 \%$ of sketches which were identified manually as 
axonometric being misclassified as central projections.

What remains unclear is whether all of these problem sketches were intended by their creators to be axonometric. Is it possible that the machine is correct, that convergence is indeed present, but that some optical illusion prevents the human eye from detecting it. This too remains a matter for future research.

\section{Acknowledgements}

The Spanish Ministry of Science and Education and the European Union (Project DPI2007-66755-C02-01), and the Ramon y Cajal Scholarship Programme are acknowledged with gratitude.

\section{References}

[1] Goel V. Sketches of Thought. The MIT Press 1995.

[2] Draper, S.W. Reasoning about Depth in LineDrawing Interpretation. PhD Thesis, Sussex University, 1980.

[3] Farish, W. On Isometrical Perspective. In: Cambridge Philosophical Transactions. 1, 1822.

[4] Biederman, I. Recognition-by-Components: A Theory of Human Image Understanding. Psychological Review, 94, 115-147, 1987.

[5] Palmer SE. Vision science. Photons to phenomenology. Cambridge, MA: The MIT Press, 1999.

[6] Hoffmann D. Visual Intelligence. How we create what we see. Norton Publishing, 1998.

[7] Sarkar S. and Boyer K.L. Perceptual Organization in Computer Vision: A review and a Proposal for a Classificatory Structure. IEEE Trans. on Systems, Man and Cybernetics, 23(2), 382 - 399, 1993.

[8] Lipson, H. and Shpitalni, M. Optimizationbased reconstruction of a $3 \mathrm{D}$ object from a single freehand line drawing. Computer-Aided Design; 28(8):651-63, 1996.

[9] Varley, P.A.C. Automatic Creation of Boundary-Representation Models from Single Line Drawings, $\mathrm{PhD}$ Thesis, University of Wales, 2003.

[10] Company, P., Contero, M., Conesa, J. and Piquer, A. An optimisation-based reconstruction engine for $3 \mathrm{D}$ modelling by sketching. Computer and Graphics 28, 955979, 2004.

[11] Yuan S., Tsui L.Y. and Jie S. Regularity selection for effective 3D object reconstruction from a single line drawing. Pattern Recognition Letters 29 (10), 14861495, 2008.

[12] Tian, C., Masry, M., Lipson, H. Physical sketching: Reconstruction and análisis of $3 \mathrm{D}$ objects from freehand sketches. Computer aided design; 41: 147-158, 2009.

[13] Parodi, P. and Torre. V. On the Complexity of Labeling Perspective Projections of Polyhedral Scenes, Artificial Intelligence 70, 239--276, 1994.

[14] Sturm. P.F. and Maybank, S.J. A Method for Interactive 3D Reconstruction of Piecewise Planar Objects from Single Images. In ed. Pridmore, A. and Elliman, D., Proc. British Machine Vision Conference, Nottingham, 265--274, 1999.

[15] Kanade, T. Recovery of the ThreeDimensional Shape of an Object from a Single View. Artificial Intelligence 17, 409-460, 1981.

[16] Prats, M., Lim, S., Jowers, I., Garner, S. \& Chase, S. Transforming shape in design: observations from studies of sketching. Design Studies 30, 503-520, 2009.

[17] Wyeld, T. The correlation between the ability to read and manually reproduce a $3 \mathrm{D}$ image: some implications for $3 \mathrm{D}$ information visualisation. $13^{\text {th }}$ International Conference Information Visualisation, 496-501, 2009.

[18] Plumed, R., Company, P., Piquer, A., Varley, P.A.C.: Convergence measure of sketched engineering drawings. Technical Report Ref. 07/2010 Regeo. Geometric Reconstruction Group, http://www.regeo.uji.es

[19] Varley, P.A.C., Martin, R.R. and Suzuki, H. Frontal Geometry from Sketches of Engineering Objects: Is Line Labelling Necessary?, Computer Aided Design 37 (12), 1285-1307, 2005 